\title{
Incubated reminiscence effects
}

\author{
STEVEN M. SMITH \\ Texas A\&M University, College Station, Texas \\ and \\ EDWARD VELA \\ California State University, Chico, California
}

\begin{abstract}
Reminiscence, the recall of material that was not successfully recalled on a previous attempt, was examined in three experiments as a function of the intertest (incubation) interval. Incubation intervals inserted between successive recall tests resulted in increased reminiscence, but the effect was seen primarily in the first retested minute. Neither the duration of the initial test (1-4 $\mathrm{min}$ ), nor the incubation activity (maze problems vs. rest) affected this incubated reminiscence effect. The results support models in which recall tests cause output interference, but incubation intervals reduce it.
\end{abstract}

When a list of items is recalled, one may experience output interference, a type of retrieval block in which memory of previously recalled information may prevent retrieval of new unrecalled material. For example, Rundus's (1973) retrieval model describes memory search as involving sampling with replacement (see also Shiffrin, 1970); retrieved items are incremented in strength and replaced within the current search set. The strengthened items thereby continue to accrue strength with subsequent retrievals, so that a point is reached in which a retrieval attempt becomes so likely to generate an already-retrieved item that as-yet-unretrieved items are almost impossible to generate. This type of model can explain the unintuitive negative effects of part-list cuing (e.g., Raaijmakers \& Shiffrin, 1981), and it provides part of an explanation of the phenomena referred to as hypermnesia and reminiscence.

When a studied list is recalled many times, it is sometimes observed that the net number recalled increases from test to test (see, e.g., Payne, 1987). This net increase constitutes hypermnesia. Reminiscence, a related phenomenon, consists of the finding that material not recalled on one test may be successfully recalled on a subsequent test. Hypermnesia results from circumstances in which the degree of reminiscence (the number of newly accessible items in memory) exceeds forgetting over time, as pointed out by Payne (1987). Thus, hypermnesia can be observed only when reminiscence occurs, and hypermnesia can never exceed reminiscence.

Although little has been said in the literature about explanations of reminiscence, one reasonable account was

The present research was supported by NIMH Grant R01 MH4473001 to Steven Smith. We wish to thank Margaret Intons-Peterson, David Payne, and Joan Snodgrass for their helpful comments on an earlier draft of the manuscript. Correspondence should be sent to Steven M. Smith, Department of Psychology, Texas A\&M University, College Station, TX 77843. offered by Madigan (1976), who hypothesized an output interference effect in immediate recall, followed by a timedependent reduction of output interference. Along the same lines of reasoning, Roediger and Thorpe (1978) suggested that Estes's (1955) stimulus sampling theory could help explain the reminiscence phenomenon. In brief, the theory states that the elements sampled from memory on two different occasions will vary, with greater variability occurring over time. Given that output interference builds up as more items are recalled, Roediger and Thorpe (1978), like Madigan (1976), suggested that reminiscence may result from a release from output interference when time passes. As time passes, it becomes more likely that new items in memory will be sampled. Since stimulus fluctuation has been used to explain forgetting, it might be said that reminiscence results when output interference is reduced by forgetting.

This explanation of output interference and reminiscence is conceptually related to the phenomena of fixation and incubation in problem solving (see, e.g., Smith \& Blankenship, 1989). Like output interference, fixation can be characterized as an inability to abandon primed information: In the case of fixation, the primed information may be an inappropriate solution. Like reminiscence, incubation represents a release from this negative priming. Smith and Blankenship (1988), who successfully induced fixation by priming inappropriate solutions to problems, found significant incubation effects only following this fixation manipulation. Using similar procedures, Smith and Blankenship (1989) found time-dependent incubation effects: Greater improvement in solving previously unsolved problems (i.e., greater incubation) was observed when retesting occurred after longer periods of incubation. These findings are consistent with the idea that as time between two work sessions on a cognitive task increases, the likelihood of searching memory differently on the two occasions also increases. The adaptive usefulness of such a pattern of accessibility depends on whether continuity 
or discontinuity will benefit the task at hand most. In the case of recovering from fixation or output interference, discontinuity is more adaptive.

Just as incubation requires time away from a problem before insight will occur, it was hypothesized that time away from a recall task would increase reminiscence and hypermnesia. Specifically, the hypothesis that motivated the present experiments states that greater "incubation" time inserted between recall tests should increase the chance of searching memory differently on the two tests, resulting in greater reminiscence.

Madigan (1976, Experiment 2) tested a similar hypothesis. Noting that studies with longer intertest intervals appeared to produce the greatest degree of reminiscence, Madigan hypothesized that temporal separation of test trials might weaken the recency bias in memory search during repeated testing, thus increasing the amount of item fluctuation within the search set between recall trials. In Experiment 2, Madigan gave three successive recall tests of a list of 36 nouns that had been studied. The tests were separated by either a 2- or a 12-min interval. Reported measures of reminiscence indicated larger effects for the 12-min condition. Furthermore, Madigan found that more reminiscence occurred early in the final recall sequence for the 12-min group than for the 2-min group. The present experiments replicate and extend these findings.

In the present study, we were also concerned with the effects of the initial recall test duration on reminiscence. Previous studies of reminiscence and hypermnesia have not been clear on the importance of this factor. Although this issue has not been systematically considered or manipulated in past studies, the pattern of allowing subjects adequate time on the initial test to recall all the items they wish to is fairly clear throughout the studies of reminiscence and hypermnesia. Most studies have allowed at least $4 \mathrm{~min}$ for the initial test, a duration long enough for subjects to recall most of the recallable items. The dilemma, however, is how to observe additional recalled material if recall attempts have already been abandoned.

Rundus (1973) postulated that after some given number of failed retrieval attempts, the subject will enforce a "stopping rule" that tells the subject to discontinue further attempts at retrieving unrecalled items. Both Rundus (1973) and Raaijmakers and Shiffrin (1981) described similar rules for abandoning retrieval cues and for abandoning the recall process. Subjects supposedly employ such rules when there appears to be little, if any, advantage to continued retrieval attempts. It should be noted that encoded target material may remain unretrieved at the time when the stopping rule is employed. Reminiscence and hypermnesia may be found if subsequent retrieval attempts occur after the accrued output interference has dissipated, when it is possible to retrieve previously unretrieved material.

It is not clear how stopping times coincide with experimenter-determined recall intervals. Is it considered reminiscence or hypermnesia if initial recall is terminated by the experimenter prior to the stopping rule's being reached? Do subjects reinitiate memory searches some time after retrieval has been stopped by the rule, thus invisibly causing the type of mnemonic incubation effect that we postulated to occur for repeated (experimenterdefined) tests? For example, in Roediger and Thorpe's (1978) 21-min recall test, subjects may have temporarily quit probing memory after a few minutes and, perhaps due to demand characteristics of the experiment, reinitiated the retrieval process later during the long test interval. Raaijmakers and Shiffrin's (1981) rechecking procedure is an example of how recall might begin again after the stopping rule has terminated the main search phase of free recall. In the present experiments, the duration of the immediate free recall test was systematically varied to better control the time initially spent searching memory.

\section{EXPERIMENT 1}

There were some unusual aspects of Madigan's (1976) experiment, in which it was found that longer intervals between tests increased reminiscence. First, although hypermnesia effects are more likely to occur for targets that are pictures rather than words (e.g., Erdelyi \& Becker, 1974; Payne, 1987), Madigan used a list of nouns. Second, there were two study and test trials prior to the final recall test, and although there was an interpolated task prior to the final recall, it is not clear to what degree subjects may have expected another test, given that repeated tests had already occurred. Thus, Madigan's subjects may have been motivated to tacitly retrieve further items during the intertest (incubation) interval. Although some (e.g., McGeoch, 1935) have found no difference in hypermnesia for subjects who did, rather than did not, covertly review list items between tests, others (e.g., Buxton, 1943) have stressed the importance of controlling and limiting such review. Third, Madigan's procedure required the subject to write each recalled word on a separate card, so that recalled words could not be reviewed, a procedure that may affect output interference. If keeping track of previously recalled items reduces output interference by deterring multiple retrievals of the items, then the lack of an opportunity to review their responses might have increased the frequency with which subjects mistakenly retrieved already-recalled items, increasing output interference. Or, if reading a target item elevates output interference, as suggested by part-list cuing studies (e.g., Slamecka, 1968), then Madigan's (1976) procedure may have decreased output interference by preventing subjects from rereading previously recalled items. It is not clear whether or not Madigan's finding depended on this unusual recall technique. Target items in the present experiments were listed by subjects on a single continuously visible page, which is more similar to typical laboratory procedures.

In Experiment 1, the generality of the finding of larger reminiscence was extended, with longer intervals between repeated recall tests. This phenomenon will be referred to as an incubated reminiscence effect. The general method 
was similar for all experiments. Subjects were presented with booklets containing pictures of common objects and were told to memorize the objects. Two free recall tests were then administered, temporally separated by either no interval or a period of incubation. The subjects were never warned of the second recall test, since we wished to minimize intentional memory searches during the intertest (incubation) intervals. Recall tests varied in duration from 1 to $4 \mathrm{~min}$, allowing a comparison between conditions in which the recallable pool of items was far from exhausted (e.g., 1-min tests) versus conditions in which initial recall was more exhausted (e.g., 4-min tests). It was predicted that, regardless of the test duration, longer intertest intervals would result in greater reminiscence and hypermnesia.

\section{Method}

Subjects. The 221 volunteers fulfilled part of a course requirement for the introductory psychology course at Texas A\&M University. The subjects in all conditions of all three experiments volunteered by signing up on posted sheets in the psychology department. Since attendance varied across the experimental sessions, there were different numbers of subjects for different experimental conditions. The numbers of subjects in each condition of Experiment 1 are shown in Table 1.

Materials. The study materials used in all the experiments of the present study consisted of a booklet with 50 line drawings taken from Snodgrass and Vanderwart (1980). Each picture was an easily labeled object. Five drawings were arranged vertically on each of 10 consecutive pages, making a total of 50 pictures. The same materials were used in all experiments.

Procedure. Each subject was given a booklet of materials and was told to memorize the list of objects for a later memory test. There were $30 \mathrm{sec}$ given to study the five pictures on each page.
A free recall test was administered immediately after the last page was studied. The subjects wrote their responses on a single blank page, and, every $60 \mathrm{sec}$, they were asked to draw a line under their most recently recalled word. At the end of the initial recall test, the subjects were asked to sit quietly while waiting for their next task. They were not told of the subsequent test, and since the single expected test had already occurred, it was assumed that they would not intentionally recall items during the incubation intervals. After the incubation interval, the subjects were given a second free recall test for the originally studied pictures. Again, the subjects marked their recall pages every $60 \mathrm{sec}$ to allow a minute-by-minute scoring of the free recall test.

Design. The duration of each of the recall tests (both initial and final recall) was 1, 2, or $4 \mathrm{~min}$, manipulated between subjects. The interval between tests, also manipulated between subjects, was 0 , 1,5 , or $10 \mathrm{~min}$. Test number was Test 1 versus retest, a withinsubjects variable. Thus, the experiment consisted of a $3 \times 4 \times 2$ (test duration $\times$ incubation interval $\times$ test number) design.

\section{Results}

Because subjects in the present experiments were selfenrolled, unequal numbers of subjects participated in the different conditions. The unequal $n s$, however, did not reflect differences in the sizes of underlying treatment populations, since the subjects were blind to the conditions in which they enrolled. Therefore, all reported ANOVAs used the method of unweighted means.

An alpha level of .05 was used for all the statistical tests reported in the present experiments.

Reminiscence. A $3 \times 4$ (test duration $\times$ incubation interval) analysis of variance (ANOVA) was computed, with number of newly recalled words at retest (i.e., reminiscence) as the dependent measure. There was no effect of test duration $\left[F(2,209)=.27, M S_{\mathrm{e}}=5.44\right]$; longer test

Table 1

Total Number Recalled, Hypermnesia, and Reminiscence as a Function of Test Duration and Incubation Interval in Experiment 1

\begin{tabular}{|c|c|c|c|c|c|c|c|c|}
\hline & \multicolumn{8}{|c|}{ Incubation Interval } \\
\hline & \multicolumn{2}{|c|}{$0 \mathrm{Min}$} & \multicolumn{2}{|c|}{$1 \mathrm{Min}$} & \multicolumn{2}{|c|}{$5 \mathrm{Min}$} & \multicolumn{2}{|c|}{$10 \mathrm{Min}$} \\
\hline & $M$ & $S D$ & $M$ & $S D$ & $M$ & $S D$ & $M$ & $S D$ \\
\hline \multicolumn{9}{|c|}{ 1-Min Test Duration } \\
\hline Total Test 1 & 14.88 & 3.41 & 15.33 & 4.35 & 13.67 & 2.54 & 16.32 & 3.58 \\
\hline Total retest & 15.00 & 3.54 & 15.95 & 3.37 & 13.50 & 2.77 & 16.79 & 3.47 \\
\hline Reminiscence & 3.12 & 2.29 & 3.86 & 2.57 & 3.00 & 1.91 & 4.95 & 2.91 \\
\hline Hypermnesia & .12 & 2.26 & .62 & 3.19 & -.17 & 2.23 & .47 & 2.63 \\
\hline$n$ & 17 & & 21 & & 18 & & 19 & \\
\hline \multicolumn{9}{|c|}{ 2-Min Test Duration } \\
\hline Total Test 1 & 19.05 & 4.82 & 18.00 & 4.07 & 15.59 & 3.06 & 16.50 & 3.55 \\
\hline Total retest & 19.47 & 5.05 & 17.53 & 4.26 & 16.06 & 3.77 & 18.30 & 4.64 \\
\hline Reminiscence & 2.63 & 2.43 & 2.53 & 1.96 & 3.71 & 1.99 & 5.15 & 2.81 \\
\hline Hypermnesia & .42 & 2.81 & -.47 & 2.80 & .47 & 2.98 & 1.80 & 3.05 \\
\hline$n$ & 19 & & 15 & & 17 & & 20 & \\
\hline \multicolumn{9}{|c|}{ 4-Min Test Duration } \\
\hline Total Test 1 & 20.37 & 6.02 & 24.33 & 6.62 & 24.21 & 4.85 & 24.32 & 5.61 \\
\hline Total retest & 20.74 & 5.94 & 25.56 & 7.35 & 25.47 & 5.02 & 26.47 & 5.55 \\
\hline Reminiscence & 2.95 & 1.84 & 2.83 & 2.04 & 4.42 & 2.12 & 4.89 & 2.58 \\
\hline Hypermnesia & .37 & 2.36 & 1.23 & 3.67 & 1.26 & 2.81 & 2.15 & 1.86 \\
\hline & 19 & & 18 & & 19 & & 19 & \\
\hline
\end{tabular}

Note-Hypermnesia $=($ retest total recall $)-($ Test 1 total recall $)$. Reminiscence $=$ Number of words recalled at retest but not on Test 1 . 
durations did not yield greater reminiscence (Table 1 ). The effect of incubation interval was significant $[F(3,209)$ $\left.=9.40, M S_{e}=5.44\right]$; there was generally greater reminiscence at longer intervals (Table 1). Newman-Keuls pairwise comparisons $(\alpha=.05)$ indicated that the 10-min incubation condition had more reminiscence than the 0 and 1-min incubation conditions, and that the 5-min incubation condition had higher reminiscence than the 1- or 0 -min conditions. No other comparisons showed significant effects.

Retest recall performance was scored in terms of the number of newly recalled items per minute (reminiscence/ minute). A newly recalled item was defined as an item not recalled on Test 1 but recalled on the retest. The minuteby-minute reminiscence results for Experiment 1 are shown in Table 2. The highest reminiscence/minute occurred in the first retested minute in the incubation conditions, but not in the immediate retest condition. The pattern of these results indicates that the positive effect of an incubation interval on reminiscence is most clearly seen in the 1 st minute of the retest.

It was possible to compare the 1st retested minute, but not later minutes of the retest, across all three test durations. A $3 \times 4$ (test duration $\times$ incubation interval) ANOVA was computed, with reminiscence for the 1 st retested minute as the dependent measure. The effect of incubation interval was significant $\left[F(3,209)=12.03, M S_{c}=3.95\right]$; reminiscence in the 1 st minute of the retest was greater following longer incubation intervals (Table 2). The effect of test duration was also significant $[F(2,209)=$ $25.73, M S_{e}=3.95$ ]; greater reminiscence was found for shorter test durations. The incubation interval $\times$ test duration interaction was not significant $[F(6,209)=1.29$, $\left.M S_{\mathrm{e}}=3.95\right]$.

To more closely examine the minute-by-minute recall results of the retest, separate ANOVAs were computed to analyze reminiscence/minute for each test duration. For the 1-min test condition, a one-way ANOVA was computed, with incubation interval as the independent vari- able and reminiscence/minute as the dependent measure. The effect of incubation interval did not quite reach significance $\left[F(3,71)=2.44, M S_{e}=6.07\right]$, although reminiscence was generally higher at the longer intervals (Table 2).

For the 2 -min test condition, a $4 \times 2$ (incubation interval $\times$ minute) ANOVA was computed, with reminiscence/ minute as the dependent measure. Minute was the 1st or 2nd minute of the retest. A significant effect of incubation interval was found $\left[F(3,67)=4.96, M S_{e}=2.79\right]$; there was generally greater reminiscence for longer intervals. There was a significant effect of minute $[F(1,67)=$ $\left.11.38, M S_{c}=2.82\right]$; more reminiscence was seen for the 1 st minute than for the 2 nd minute of the retest. The incubation interval $\times$ minute interaction was significant $\left[F(3,67)=5.36, M S_{\mathrm{e}}=2.82\right] ;$ longer intervals yielded greater reminiscence in the 1 st retested minute, but not in the 2nd minute (Table 2).

For the 4-min test condition, a $4 \times 4$ (incubation interval $\times$ minute) ANOVA was computed, with reminiscence/ minute as the dependent measure. Minute was the 1st, 2nd, 3rd, or 4th minute of the retest. A significant effect of incubation interval was found $\left[F(1,71)=4.33, M S_{\mathrm{e}}=\right.$ 1.17]; again, there was greater reminiscence/minute for longer intervals. The effect of minute was significant $\left[F(3,213)=6.98, M S_{\mathrm{e}}=1.33\right]$; greater reminiscence was fund in the 1st minute of the retest than in the later minutes. The incubation interval $\times$ minute interaction was also significant $\left[F(9,213)=2.69, M S_{\mathrm{e}}=1.33\right]$; again, there was greater reminiscence at longer intervals for the 1 st retested minute, but not for other minutes (Table 2).

Hypermnesia. A $3 \times 4 \times 2$ (test duration $\times$ incubation interval $\times$ test number) ANOVA was computed, with total number of words recalled as the dependent measure. The effect of test number was significant $[F(1,209)=$ 13.65, $\left.M S_{\mathrm{e}}=3.82\right]$. As the means in Table 1 indicate, there was a hypermnesia effect, or a net increase in recall from Test 1 to the retest. The test duration $\times$ test number interaction did not reach significance $[F(2,209)=$

Table 2

Minute-by-Minute Reminiscence as a Function of Incubation Interval and Test Duration in Experiment 1

\begin{tabular}{|c|c|c|c|c|c|c|c|c|}
\hline & \multicolumn{8}{|c|}{ Incubation Interval } \\
\hline & \multicolumn{2}{|c|}{$0 \mathrm{Min}$} & \multicolumn{2}{|c|}{$1 \mathrm{Min}$} & \multicolumn{2}{|c|}{$5 \mathrm{Min}$} & \multicolumn{2}{|c|}{$10 \mathrm{Min}$} \\
\hline & $M$ & $S D$ & $M$ & $S D$ & $M$ & $S D$ & $M$ & $S D$ \\
\hline \multicolumn{9}{|c|}{ 1-Min Test Duration } \\
\hline 1st minute & 3.11 & 2.29 & 3.86 & 2.57 & 3.00 & 1.91 & 4.95 & 2.91 \\
\hline \multicolumn{9}{|c|}{ 2-Min Test Duration } \\
\hline 1st minute & 1.21 & 1.62 & 1.27 & 1.28 & 2.65 & 1.77 & 3.80 & 2.33 \\
\hline 2nd minute & 1.42 & 1.35 & 1.27 & 1.39 & 1.06 & 1.20 & 1.35 & 1.93 \\
\hline \multicolumn{9}{|c|}{ 4-Min Test Duration } \\
\hline 1st minute & .63 & .76 & .83 & 1.15 & 1.79 & 1.40 & 2.47 & 2.39 \\
\hline 2nd minute & .42 & .61 & .56 & .70 & .90 & .94 & .79 & .79 \\
\hline 3rd minute & .79 & .79 & .78 & 1.11 & .58 & .69 & .68 & .75 \\
\hline 4th minute & 1.11 & 1.41 & .67 & 1.14 & 1.16 & 1.17 & .95 & 1.08 \\
\hline
\end{tabular}

Note-Reminiscence $=$ Number of words recalled (in one minute) at retest but not on Test 1. 
2.53, $M S_{\mathrm{e}}=3.82$ ], although Table 1 shows a trend suggesting greater hypermnesia for the longer test durations. The test number $X$ incubation interval interaction also did not reach significance $\left[F(3,209)=2.11, M S_{\mathrm{e}}=3.82\right]$, although a trend suggesting greater hypermnesia for longer incubation intervals can be seen in Table 1 .

\section{Discussion}

The experimental predictions were partially supported by the results. Reminiscence was increased when incubation intervals were interposed between Test 1 and the retest, an effect that appeared to hold regardless of the test duration. This finding both replicates and extends Madigan's (1976) results, and it supports the idea of a timerelated reduction in output interference. Hypermnesia, a net increase in recall, followed a similar trend, but it was not significantly affected by incubation intervals.

The clearest effect of incubation intervals was seen in the first minute of the retest. Most of the observed reminiscence occurred in the first retested minute, in which the number of newly recalled items was reliably related to the incubation interval. Reminiscence after the first minute did not show such a pattern. These results are consistent with the theory that fluctuation of stimulus elements occurred in the incubation interval, causing subjects to probe memory at retest more variably than if retesting had occurred immediately. The set of elements used to probe memory following the ist retested minute may have stabilized, so that output interference and priming from the 1st minute had a much greater effect than the preceding incubation interval on subsequent minutes of the retest.

Although there were highly reliable effects of test duration on reminiscence in the early minutes of the retest, this variable did not interact with interval. Incubated reminiscence effects were found for all three studied test durations. Assuming that longer test durations decrease the pool of initially inaccessible but available items, these findings indicate that incubated reminiscence does not necessarily depend on the size of this pool.

\section{EXPERIMENT 2}

Because reminiscence/incubation effects could not be compared across test durations after the 1 st minute of the retest in Experiment 1, the retest durations were made an equal $5 \mathrm{~min}$ for all subjects in Experiment 2. Thus, it could be determined whether or not the incubated reminiscence effects were confined to the first retested minute for all initial test durations, as in Experiment 1.

\section{Method}

Subjects. The 233 volunteers who participated in Experiment 2 fulfilled part of a course requirement for the introductory psychology course at Texas A\&M University. The numbers of subjects in each experimental condition are shown in Table 3.

Design and Procedure. For the incubation groups, the materials, design, and procedure were the same as in Experiment 1, except that the retest was $5 \mathrm{~min}$ in duration for all subjects in all groups.

\section{Results}

The analyses in Experiment 2 were the same as those in Experiment 1, except that all 4 min of the retest could be compared across conditions, because all subjects in Experiment 2 had 4-min retests.

Reminiscence. A $3 \times 4$ (Test 1 duration $\times$ incubation interval) ANOVA was computed, with reminiscence (total number of newly recalled words at retest) as the de-

Table 3

Total Number Recalled, Hypermnesia, and Reminiscence as a Function of Test Duration and Incubation Interval in Experiment 2

\begin{tabular}{|c|c|c|c|c|c|c|c|c|}
\hline & \multicolumn{8}{|c|}{ Incubation Interval } \\
\hline & \multicolumn{2}{|c|}{$0 \mathrm{Min}$} & \multicolumn{2}{|c|}{$1 \mathrm{Min}$} & \multicolumn{2}{|c|}{$5 \mathrm{Min}$} & \multicolumn{2}{|c|}{$10 \mathrm{Min}$} \\
\hline & $M$ & $S D$ & $M$ & $S D$ & $M$ & $S D$ & $M$ & $S D$ \\
\hline \multicolumn{9}{|c|}{ 1-Min Test Duration } \\
\hline Total Test 1 & 13.63 & 3.96 & 13.56 & 3.39 & 16.14 & 3.55 & 15.11 & 3.53 \\
\hline Total retest & 21.44 & 7.04 & 20.44 & 6.86 & 23.86 & 6.29 & 23.50 & 5.43 \\
\hline Reminiscence & 10.13 & 5.21 & 8.25 & 4.52 & 9.10 & 4.33 & 10.56 & 3.40 \\
\hline Hypermnesia & 7.81 & 7.26 & 6.88 & 4.62 & 7.72 & 5.05 & 8.39 & 3.65 \\
\hline$n$ & 16 & & 16 & & 21 & & 18 & \\
\hline \multicolumn{9}{|c|}{ 2-Min Test Duration } \\
\hline Total Test 1 & 19.89 & 6.32 & 17.32 & 4.74 & 16.82 & 4.85 & 18.77 & 5.02 \\
\hline Total retest & 24.61 & 5.09 & 23.00 & 7.05 & 21.71 & 5.98 & 22.69 & 7.04 \\
\hline Reminiscence & 6.06 & 2.53 & 7.24 & 3.60 & 6.00 & 3.24 & 7.00 & 3.83 \\
\hline Hypermnesia & 4.72 & 2.87 & 5.68 & 3.68 & 4.89 & 3.71 & 3.92 & 4.21 \\
\hline$n$ & 18 & & 25 & & 17 & & 13 & \\
\hline \multicolumn{9}{|c|}{ 4-Min Test Duration } \\
\hline Total Test 1 & 22.80 & 4.49 & 23.60 & 7.39 & 21.27 & 4.85 & 21.19 & 4.53 \\
\hline Total retest & 24.36 & 5.83 & 24.47 & 9.05 & 24.32 & 6.63 & 23.30 & 6.66 \\
\hline Reminiscence & 4.44 & 2.24 & 4.33 & 2.87 & 5.23 & 2.69 & 4.89 & 2.69 \\
\hline Hypermnesia & 1.56 & 3.00 & .87 & 4.55 & 3.05 & 3.20 & 2.11 & 4.24 \\
\hline$n$ & 25 & & 15 & & 22 & & 27 & \\
\hline
\end{tabular}

Note - Hypermnesia $=($ retest total recall $)-($ Test 1 total recall $)$. Reminiscence $=$ Number of words recalled at retest but not on Test 1 . 
pendent measure. Only Test 1 duration had a significant effect $\left[F(2,221)=37.12, M S_{c}=11.88\right]$; greater reminiscence was found for shorter Test 1 durations (Table 3 ). Neither the main effect of incubation interval nor the incubation interval $\times$ Test 1 duration interaction was significant (both $F \mathrm{~s}<1.0, M S_{\mathrm{e}}=11.88$ ).

As in Experiment 1, retest recall performance was scored in terms of the number of newly recalled items per minute (reminiscence/minute). The minute-by-minute recall results for Experiment 2 are shown in Table 4 . The pattern of these results indicates that the position effect of an incubation interval on reminiscence is again clearly seen only in the 1st minute of the retest.

A $3 \times 4 \times 5$ (Test 1 duration $x$ incubation interval $\times$ minute) ANOVA was computed, with reminiscence/ minute as the dependent measure, with minute referring to the 1st, 2nd, 3rd, 4 th, or 5th minute of the retest. The significant effect of minute $\left[F(4,884)=67.19, M S_{\mathrm{e}}=\right.$ 2.07] was due to diminished reminiscence/minute for successive minutes of the retest (Table 4). A significant interaction of incubation interval $\times$ minute $[F(12,884)=4.96$, $\left.M S_{\mathrm{e}}=2.07\right]$ appears to have resulted from greater reminiscence at longer incubation intervals for the first retested minute, but not for subsequent minutes of the retest (Table 6). Newman-Keuls pairwise comparisons showed that reminiscence for the 1st retested minute was significantly greater in the incubation conditions than in the no incubation condition $(\alpha=.01)$, and that the three incubation groups did not differ from each other. Furthermore, reminiscence in the 1 st retested minute exceeded reminiscence in the other retested minutes for all of the incubation groups, but not for the no incubation groups.
Hypermnesia. A $3 \times 4 \times 2$ (Test 1 duration $\times$ incubation interval $\times$ test number) ANOVA was computed, with total number of words recalled as the dependent measure. Test 1 duration was 1,2 , or $4 \mathrm{~min}$, and incubation interval was $0,1,5$, or $10 \mathrm{~min}$. Test number was Test 1 versus retest, a within-subjects variable. The effect of test number was significant $\left[F(1,221)=287.31, M S_{c}=8.90\right]$; as indicated by Table 3, there was a reliable hypermnesia effect. Greater hypermnesia occurred for the briefer Test 1 conditions; there was a significant Test 1 duration $\times$ test number interaction $\left[F(2,221)=36.37, M S_{e}=\right.$ 8.90]. The test number $\times$ incubation interval interaction was not significant $(F<1.0)$.

\section{Discussion}

Although the overall reminiscence and hypermnesia effects, totaled across all $5 \mathrm{~min}$ of the retest, did not show significant effects of incubation intervals, the pattern of results is similar to that found in Experiment 1. Incubated reminiscence effects showed up clearly in the 1st retested minute, and the effects were not apparent after the 1st retested minute. These results are consistent with the hypothesis that longer intervals increased the variability in sampling the available memory set. After a minute of recalling information, output interference apparently increased enough to limit reminiscence regardless of the incubation interval.

Reminiscence and hypermnesia were decreased by longer Test 1 durations, indicating that the set of available but initially inaccessible items was greater following briefer initial recall tests. Incubated reminiscence effects (in the 1st retested minute), however, were found

Table 4

Minute-by-Minute Reminiscence as a Function of Incubation Interval and Test Duration in Experiment 2

\begin{tabular}{|c|c|c|c|c|c|c|c|c|}
\hline & \multicolumn{8}{|c|}{ Incubation Interval } \\
\hline & \multicolumn{2}{|c|}{0 Min } & \multicolumn{2}{|c|}{1 Min } & \multicolumn{2}{|c|}{5 Min } & \multicolumn{2}{|c|}{$10 \mathrm{Min}$} \\
\hline & $M$ & $S D$ & $M$ & $S D$ & $M$ & $S D$ & $M$ & $S D$ \\
\hline \multicolumn{9}{|c|}{ 1-Min Test Duration } \\
\hline Ist minute & 2.75 & 2.35 & 3.75 & 2.05 & 4.81 & 2.58 & 5.39 & 2.66 \\
\hline 2nd minute & 2.44 & 2.25 & 1.81 & 1.64 & 1.52 & 1.40 & 2.17 & 2.15 \\
\hline 3rd minute & 2.19 & 1.52 & 1.38 & 1.02 & 1.10 & 1.30 & 1.44 & 1.15 \\
\hline 4th minute & 1.13 & 1.02 & 1.00 & 1.59 & 1.14 & 1.59 & .78 & .65 \\
\hline 5 th minute & 1.63 & 1.20 & .31 & .70 & .52 & 1.17 & .78 & .94 \\
\hline \multicolumn{9}{|c|}{ 2nd-Min Test Duration } \\
\hline 1st minute & 1.72 & 2.16 & 2.96 & 2.24 & 2.29 & 2.14 & 3.23 & 2.83 \\
\hline 2nd minute & 1.39 & 1.38 & 1.72 & 2.01 & 1.47 & 1.55 & 1.08 & 1.44 \\
\hline 3rd minute & 1.06 & 1.21 & .96 & 1.10 & 1.06 & 1.20 & 1.08 & 1.61 \\
\hline 4th minute & 1.33 & 1.33 & .84 & 1.11 & .76 & 1.09 & 1.00 & 1.29 \\
\hline 5th minute & .56 & .78 & .76 & 1.05 & .41 & .62 & .62 & .96 \\
\hline \multicolumn{9}{|c|}{ 4-Min Test Duration } \\
\hline 1st minute & .60 & .82 & 1.13 & 1.13 & 2.18 & 2.13 & 1.89 & 1.60 \\
\hline 2nd minute & .64 & .91 & .87 & .99 & .68 & 1.04 & .92 & 1.17 \\
\hline 3rd minute & 1.04 & 1.24 & 1.07 & 1.16 & .77 & 1.45 & .52 & .64 \\
\hline 4th minute & 1.12 & 1.13 & .80 & 1.32 & 1.14 & 1.25 & .93 & .96 \\
\hline 5th minute & 1.04 & 1.46 & .47 & .83 & .45 & .91 & .63 & .88 \\
\hline
\end{tabular}

Note-Reminiscence $=$ Number of words recalled (in $1 \mathrm{~min}$ ) at retest but not on Test 1 . 
for all initial test durations. Reaching a stopping rule (see, e.g., Rundus, 1973), then, is apparently not necessary for observing such effects. Subjects were still recalling items after a 1-min test, as is evidenced by the finding that more Test 1 items were recalled by subjects with more than a minute for the initial test (Table 3 ).

The results of Experiments 1 and 2 may be interpreted in support of the hypothesis that incubation time increased fluctuation in the stimulus set, causing greater reminiscence, at least at the beginning of a retest. This is because the memory probes initiating Test 1 and retest retrievals should be more similar when the retest is immediate than when incubation time is interposed between the two recall tests. The results are also consistent with the hypothesis that initial output interference wears off in a time-dependent manner, thus diminishing the temporary retrieval blocks acquired during Test 1 retrievals.

Both of these interpretations, however, are different from the hypothesis that subjects may have spent the incubation time recalling new items not recalled on the first test. If this were the case, subjects might begin the retest by writing down the items recalled during the incubation interval, thus causing the incubated reminiscence observed in the 1st retested minute. Roediger and Thorpe (1978), for example, found that recall can continue to improve if the subject is given extended recall time. This extra recall during the incubation interval may have occurred in Madigan's (1976) study, because there were three successive recall tests. Once they had their first retest, Madigan's subjects may have suspected that still another test would be given. In Experiments 1 and 2 of the present study, there was only one unannounced retest; thus, we assumed that the lack of an expectation of a retest would minimize recall attempts during the incubation period. It is possible, however, that subjects spontaneously recalled items during the incubation intervals. Therefore, in Experiment 3 , the opportunity for recall practice during the incubation intervals was varied.

\section{EXPERIMENT 3}

The question of tacit retrieval during the incubation interval was addressed in Experiment 3. Whether prevention of covert review of list items between tests is an important control for observing reminiscence, as suggested by Buxton (1943), or whether such review has little effect on reminiscence, as reported by McGeoch (1935), we nonetheless wished to examine recall following a period of time in which subjects were not engaged in either covert or overt retrieval of list items. This was because the present experiments were focused on incubation effects, with reminiscence as evidence of a type of incubation effect. Incubation implies that subjects are not consciously working on the critical task for a period of time.

In one condition of Experiment 3, demanding tasks were inserted in the incubation interval. Given that concurrent tasks occupy attentional capacity, it was assumed that the demanding tasks between tests would reduce the subject's opportunity to retrieve material between the tests. If tacit retrieval is responsible for the incubated reminiscence effects seen in the first two experiments, then inserting these tasks between recall tests should diminish the effects.

Alternatively, it may be that incubated reminiscence effects are due to fluctuations of the active stimulus elements between tests (Estes, 1955; Madigan, 1976; Roediger \& Thorpe, 1978) that allow memory to be probed by a different set of elements at retest. If so, tasks inserted between successive recall tests may increase the fluctuation of stimulus elements and therefore might enhance the incubated reminiscence effect. Thus, the tacit retrieval hypothesis predicts that an unfilled interval should lead to greater reminiscence effects than a filled interval, whereas the fluctuation hypothesis predicts that a filled interval will increase reminiscence and hypermnesia relative to an unfilled incubation condition.

It was also predicted that incubated reminiscence effects would appear in the 1st retested minute, as in Experiments 1 and 2 .

\section{Method}

Subjects. The 154 volunteers who participated in Experiment 3 fulfilled part of a requirement for introductory psychology at Texas A\&M University. The number of subjects in each condition is shown in Table 5.

Design and Procedure. The procedures for the study and test phases of Experiment 3 were essentially the same as those described for Experiment 1. In all conditions of Experiment 3, however, the first free recall test lasted $3 \mathrm{~min}$, and the second test was $5 \mathrm{~min}$ in duration. The subjects were not informed in advance that there would be a second recall test.

The two recall tests were separated by no interval, by $10 \mathrm{~min}$ of quiet time, or by $10 \mathrm{~min}$ of work on difficult maze problems. These three group conditions were called the no incubation, unfilled incubation, and filled incubation groups, respectively. With the exceptions noted above, the no incubation group was treated exactly as described for the 0 -min incubation groups in Experiments 1 and 2. The unfilled incubation group likewise corresponded to the 10-min incubation groups in Experiments 1 and 2. The filled incubation group was given a page with two very complex mazes. They were given 10 min to work on the mazes. No subjects completed both mazes in the time given. The recall test was given after the maze task.

Table 5

Total Number Recalled, Hypermnesia, and Reminiscence as a Function of Condition in Experiment 3

\begin{tabular}{|c|c|c|c|c|c|c|}
\hline & \multicolumn{6}{|c|}{ Condition } \\
\hline & \multicolumn{2}{|c|}{$\begin{array}{c}\text { No } \\
\text { Incubation }\end{array}$} & \multicolumn{2}{|c|}{$\begin{array}{l}\text { Unfilled } \\
\text { Incubation }\end{array}$} & \multicolumn{2}{|c|}{$\begin{array}{c}\text { Filled } \\
\text { Incubation }\end{array}$} \\
\hline & $M$ & $S D$ & $M$ & $S D$ & $M$ & $S D$ \\
\hline Total Test 1 & 21.09 & 5.56 & 20.60 & 5.43 & 20.35 & 5.59 \\
\hline Total retest & 23.25 & 6.47 & 23.04 & 5.95 & 23.92 & 6.72 \\
\hline Reminiscence & 4.00 & 2.60 & 4.91 & 2.43 & 5.50 & 3.10 \\
\hline Hypermnesia & 2.16 & 2.98 & 2.44 & 3.03 & 3.57 & 3.37 \\
\hline$n$ & 57 & & 45 & & 52 & \\
\hline
\end{tabular}

Note-Hypermnesia $=$ (retest total recall) - (Test 1 total recall). Reminiscence $=$ Number of words recalled at retest but not on Test 1 . 


\section{Results}

Reminiscence. A one-way ANOVA was computed to compare the three conditions (no incubation, unfilled incubation, and filled incubation), with reminiscence (total number of newly recalled words at retest) as the dependent measure. The effect of condition was significant $\left[F(2,151)=4.18, M S_{\mathrm{e}}=7.46\right]$. Newman-Keuls pairwise comparisons $(\alpha=.05)$ indicated that reminiscence was greater in the filled incubation condition than the no incubation condition (critical difference $=1.27$ ). The unfilled incubation condition did not significantly differ from either of the other two conditions (Table 5).

Retest recall performance was scored in terms of the number of newly recalled items per minute (reminiscence/ minute). The minute-by-minute recall results for Experiment 3 are shown in Table 6 . The pattern of these results indicates that the positive effect of an incubation interval on reminiscence is again clearly seen only in the 1st minute of the retest.

A $3 \times 5$ (condition $\times$ minute) ANOVA was computed, with reminiscence/minute as the dependent measure, and with minute referring to the $1 \mathrm{st}$, 2nd, 3rd, 4th, or 5th minute of the retest. The effect of condition was significant $\left[F(2,151)=4.18, M S_{\mathrm{e}}=1.49\right]$; the least reminiscence occurred for the no incubation condition, and the most reminiscence was seen in the filled incubation condition. The effect of minute was significant $[F(4,604)=$ 9.73, $M S_{\mathrm{e}}=1.30$ ]: less reminiscence occurred for successive minutes of the retest (Figure 5).

The condition $\times$ minute interaction was significant $\left[F(8,604)=2.73, M S_{\mathrm{e}}=1.30\right]$. Newman-Keuls pairwise comparisons $(\alpha=.01)$ indicated that reminiscence was greater (by 1.016) in the first retested minute of the filled incubation condition than in the first retested minute of the no incubation condition (critical difference $=.848$ ). The unfilled incubation group had a higher reminiscence score in the 1st retested minute than the no incubation group did, but the difference (.628) did not reach significance (critical difference $=.715$ for $\alpha=.05$ ).

None of the other retested minutes showed the same pattern as did the 1 st retested minute.

Hypermnesia. A $3 \times 2$ (condition $\times$ test) ANOVA was computed, with total number of words recalled as the de-

Table 6

Minute-by-Minute Reminiscence as a Function of Condition in Experiment 3

\begin{tabular}{|c|c|c|c|c|c|c|}
\hline & \multicolumn{6}{|c|}{ Condition } \\
\hline & \multicolumn{2}{|c|}{$\begin{array}{c}\text { No } \\
\text { Incubation }\end{array}$} & \multicolumn{2}{|c|}{$\begin{array}{c}\text { Unfilled } \\
\text { Incubation }\end{array}$} & \multicolumn{2}{|c|}{$\begin{array}{c}\text { Filled } \\
\text { Incubation }\end{array}$} \\
\hline & $M$ & $S D$ & $M$ & $S D$ & $M$ & $S D$ \\
\hline 1st minute & .77 & .82 & 1.40 & 1.66 & 1.79 & 1.51 \\
\hline 2nd minute & .82 & 1.12 & 1.13 & 1.12 & 1.31 & 1.44 \\
\hline 3rd minute & .88 & 1.09 & 1.07 & 1.07 & 1.21 & 1.47 \\
\hline 4th minute & .91 & .89 & .69 & .90 & .63 & .95 \\
\hline 5th minute & .61 & 1.05 & .62 & .78 & .56 & .87 \\
\hline
\end{tabular}

Note-Reminiscence $=$ Number of words recalled (in $1 \mathrm{~min}$ ) at retest but not on Test 1. pendent measure. Condition, a between-subjects variable, consisted of no incubation, unfilled incubation, or filled incubation. Test was Test 1 versus retest, a within-subjects variable. The effect of test was significant $[F(1,151)=$ $\left.115.43, M S_{\mathrm{e}}=4.91\right]$; there was a reliable hypermnesia effect (Table 5). The interaction of condition with test number was marginally significant $[F(2,151)=3.03$, $\left.M S_{\mathrm{e}}=66.85\right]$. Although the effect was not significant, net recall increases appeared to be greatest in the filled incubation condition and smallest in the no incubation condition (Table 5).

\section{Discussion}

Experiment 3 provides another finding of incubated reminiscence effects, again demonstrating that the effects occur primarily in the 1st retested minute. The incubated reminiscence effect in Experiment 3 was at least as great in the filled condition as in the unfilled condition. Since hypermnesia and reminiscence were not enhanced by providing an opportunity for retrieval practice in the incubation interval (in the unfilled condition), it is reasonable to conclude that the effects of incubation in the present experiments were not due to increased opportunity for retrieval practice.

In fact, the trend suggests that hypermnesia may be enhanced, rather than decreased, by distractor activity interpolated between recall tests. The distractor may serve to further increase the variability with which the elements of memory are sampled on the two tests. The present experiments, however, do not directly address this issue of increased variability, and the benefit of the interpolated distractor was not significant; thus, the hypothetical increased variability caused by the distractor activity was not demonstrated by the results of Experiment 3 .

\section{GENERAL DISCUSSION}

The three experiments in the present study consistently support the idea that incubated reminiscence effects can be reliably found, although measures of total reminiscence and hypermnesia may not always show the effects. The effect of incubation on hypermnesia was marginal in Experiments 1 and 3, and not significant in Experiment 2. Incubation reliably affected total reminiscence in Experiments 1 and 3, but not in Experiment 2. The most reliable findings of incubated reminiscence occurred in the lst retested minute; later retested minutes were generally insensitive to effects of incubation intervals. The effect of incubation in the 1st retested minute was statistically reliable in all three experiments, even in Experiment 2, in which overall measures of reminiscence and hypermnesia did not reveal incubation effects.

Why were the observed incubated reminiscence effects confined to the 1 st retested minute? The present experiments did not address this question, but we will offer some possibilities. One is that output interference accrued quickly during the retest, limiting subsequent recall to "stronger" items, such as those previously recalled (on 
Test 1). A second hypothesis is that the increased retention interval in the incubation conditions caused timedependent forgetting, which counteracted the benefits of the incubation interval. That is, the interval may decrease retention overall, but still increase reminiscence in the $1 \mathrm{st}$ retested minute by momentarily decreasing output interference from Test 1 . Further study of the phenomenon may test these hypotheses.

The present findings indicate that incubated reminiscence occurs even following an initial test in which recall is terminated well before memory of the targets is exhausted. That initial recall was greater for longer tests is not surprising, and it verifies that target memory was not exhausted (i.e., the stopping rule criterion had not been reached) when the experimenter terminated the initial recall test for brief test durations. That the same incubated reminiscence effects can be seen for all initial test durations shows that such exhaustion may not be required for the effects to be observed.

It is possible, of course, that in conditions in which Test 1 was very brief (i.e., $1-\mathrm{min}$, and perhaps even 2 min, test conditions), the subjects did not have enough time to write down all the items they implicitly retrieved. Such items would show up as reminiscence when written down on the retest, even though they had actually been retrieved on Test 1 . This possibility does not seriously challenge the interpretation of the results, however, for two reasons. First, the difference in the recall of such putative items between immediate and delayed retest conditions should still reflect differences in the way subjects search memory in those conditions. Second, the occurrence of such "retrieved but unwritten items" would have been very unlikely to occur in the 4-min Test 1 conditions.

The incubated reminiscence effect may be a useful method for studying other incubation effects in the laboratory. Woodworth and Schlosberg (1954), in a description of incubation in problem solving, stated, "When the thinker makes a false start, he slides insensibly into a groove and may not be able to escape at the moment," and then, "the incubation period simply allows time for an erroneous set to die out and leave the thinker free to take a fresh look at his problem"' (p. 841). This description of fixation and incubation is very similar to descriptions of output interference and reminiscence (see, e.g., Rundus, 1973; Madigan, 1976; Roediger \& Thorpe, 1978). Thus, the reminiscence paradigm used in the present experiments may provide another method for investigating incubation effects. The study of incubation in problem solving eluded investigation until recently, because reliable techniques for observing the phenomenon in the laboratory had not been discovered. The most successful methods for observing incubation have been to approach the phenomenon as a memory-accessibility issue (Smith \& Blankenship, 1988, 1989; Yaniv \& Meyer, 1987), as in the present study.

One hypothesis to account for incubation in problem solving is that extra work may be devoted to the unsolved problem during the incubation interval, when the subject is supposed to have put the problem aside. This extra work hypothesis was tested in the incubated reminiscence paradigm in Experiment 3. Filling the incubation interval with a demanding irrelevant task, which should have decreased extra work in the incubation interval, did not decrease the incubated reminiscence effect. If anything, the filled interval resulted in more incubation, although the effect was not significant. A contextual fluctuation model (e.g., Bower, 1972; Mensink \& Raaijmakers, 1989) should, in fact, predict that certain activities in the incubation interval will increase contextual fluctuation and therefore increase incubation effects. Future research may discover whether there are incubation tasks or activities that enhance incubation, or whether the passage of time alone leads to incubation.

\section{REFERENCES}

Bower, G. H. (1972). Stimulus-sampling theory of encoding variability. In A. W. Melton \& E. Martin (Eds.), Coding processes in human memory (pp. 85-123). Washington, DC: Winston.

Buxton, C. E. (1943). The status of research in reminiscence. Psychological Bulletin, 40, 313-340.

ERdelyi, M. H., \& Becker, J. (1974). Hypermnesia for pictures: Incremental memory for pictures but not for words in multiple recall trials. Cognitive Psychology, 6, 159-171.

ESTES, W. K. (1955). Statistical theory of spontaneous recovery and regression. Psychological Review, 62, 145-154.

Madigan, S. (1976). Reminiscence and item recovery in free recall. Memory \& Cognition, 4, 233-236.

McGeoch, G. O. (1935). The conditions of reminiscence. American Journal of Psychology, 47, 65-89.

Mensink, G., \& Ranijmakers, J. G. W. (1989). A model for interference and forgetting. Psychological Review, 95, 434-455.

PAYNE, D. G. (1987). Hypermnesia and reminiscence in recall: A historical and empirical review. Psychological Bulletin, 101, 5-27.

RaAiJmakers, J. G. W., \&hiffrin, R. M. (1981). Search of associative memory. Psychological Review, 88, 93-134

RoEDiger, H. L., III, \& ThORPE, L. A. (1978). The role of recall time in producing hypermnesia. Memory \& Cognition, 6, 296-305.

Rundus, D. (1973). Negative effects of using list items as recall cues. Journal of Verbal Learning \& Verbal Behavior, 12, 43-50.

Shiffrin, R. M. (1970). Memory search. In D. A. Norman (Ed.), Models of human memory (pp. 375-447). New York: Academic Press.

SlameCKa, N. J. (1968). An examination of trace storage in free recall. Journal of Experimental Psychology, 76, 504-513.

Smith, S. M., \& Blankenship, S. E. (1988, November). An accessibility interpretation of fuxation and incubation. Paper presented at the annual meeting of the Psychonomic Society, Chicago.

SMith, S. M., BLANKenShIP, S. E. (1989). Incubation effects. Bulletin of the Psychonomic Society, 27, 311-314.

Snodgrass, J. G., Vanderwart, M. (1980). A standardized set of 260 pictures: Norms for name agreement, image agreement, familiarity, and visual complexity. Journal of Experimental Psychology: $\mathrm{Hu}$ man Learning \& Memory, 6, 174-215.

WoODWORTh, R. S., SChLOSBERG, H. (1954). Experimental Psychology: Revised Edition. New York: Holt, Rhinehart \& Winston.

YanIV, I. , Meyer, D. E. (1987). Activation and metacognition of inaccessible stored information: Potential bases for incubation effects in problem solving. Journal of Experimental Psychology: Learning, Memory, \& Cognition, 13, 187-205.

(Manuscript received November 29, 1989; revision accepted for publication June 29, 1990.) 